\title{
FIGO Stage IIA2
}

National Cancer Institute

\section{Source}

National Cancer Institute. FlGO Stage IIA2. NCI Thesaurus. Code C162224.

A FIGO stage term that applies to gynecologic cancers. For cervical cancer, it refers to cancer limited to the upper two-thirds of the vagina without parametrial involvement, equal or more than $4 \mathrm{~cm}$ in greatest dimension. 